\title{
Pedestrian dynamics with mechanisms of anticipation and attraction
}

\author{
Yi-Xuan Lü $\odot$, Zhi-Xi Wu, ${ }^{*}$ and Jian-Yue Guan \\ Institute of Computational Physics and Complex Systems, Lanzhou University, Lanzhou, Gansu 730000, China
}

(Received 2 May 2020; accepted 20 October 2020; published 18 November 2020)

\begin{abstract}
We study the pedestrian dynamics with the consideration of two heuristic mechanisms of anticipation and attraction. The former enables the individuals to anticipate potential collisions in terms of the state of surrounding environment and slow down their moving speed accordingly, while the latter reflects the tendency of the individuals following (or being attracted by) those peer partners with high moving speeds, trying to reach their destinations as quickly as possible. By tuning the interaction ranges of anticipation and attraction, we explore the collection motion behavior of the pedestrians in three common movement scenarios, i.e., the bidirectional pedestrian flows in a straight corridor, the crossing pedestrian flows in a square and the crowd evacuation in a room. Interestingly, with appropriate combinations of the anticipation distance and attraction distance, we reproduce the typical collective motion patterns of the pedestrians observed in the empirical studies, such as the formation and separation of global pedestrian flow lanes, the stop-and-go waves, and the vortexlike patterns as well. Particularly, we find that a strong attraction effect by the fast-moving pedestrian flows benefits the efficient passing only in the corridor case, whereas in the case of crossing pedestrians the avoidance behavior due to anticipation will play a dominating role in guaranteeing an ordered collective motion.
\end{abstract}

DOI: 10.1103/PhysRevResearch.2.043250

\section{INTRODUCTION}

Collective ordered motion with distinct patterns are discovered in many real-life situations, ranging from the organization of cells [1], migration of animals [2] to the traffic of human beings [3], etc. Specifically, it was observed in these multibody systems that simple local-scale interactions among individuals usually lead to complex structures macroscopically, such like vortex in sardines [4], marching queues in spiny lobsters [5,6], and lanes in pedestrians [7,8]. In the last decades, a lot of insightful models have been introduced to reproduce and explain the emergence of collective motion behavior of animals as well as our human beings [9-11]. It is worth pointing out that the occurrence of ordered collective motion is identified as a nonequilibrium phase transition from the viewpoint of statistical mechanics, and thereby the ideas and methods from the field of statistical physics have been extensively utilized to treat the collection motion phenomena $[11,12]$.

As a major research area for collective motion, pedestrian dynamics in confined space is quite interesting for scientists [13]. Particularly, when the population density becomes sufficiently large, crises and disasters may happen occasionally in human crowds [14], resulting in great economic and life loss. Therefore one of the important aims of studying

\footnotetext{
*wuzhx@1zu.edu.cn

Published by the American Physical Society under the terms of the Creative Commons Attribution 4.0 International license. Further distribution of this work must maintain attribution to the author(s) and the published article's title, journal citation, and DOI.
}

pedestrian dynamics is trying to find potential interventions to prevent accidents to arise $[15,16]$. Noticeably, thanks to the development of data analysis technique, individual recognition and tracer technique in images in recent years, more and more empirical data of real social events become available, which provide us practical views on understanding the quantitative relationship between relative position and velocity of the pedestrians and the emergent collective motion pattern $[7,8,14,16-20]$.

In theoretical studies of the pedestrian dynamics, researchers have constructed various dynamical models to depict different situations that people may face [21]. To the best of our knowledge, most existing models in the literature are either based on the fluid dynamics, which regards pedestrians as continuum matter [22-26], or on cellular automaton [27-29] and multi-agent-based models, which regards pedestrians as active agents (or particles) [30-32]. In the latter case, the pedestrian dynamics is usually studied within the framework of the so-called social force model, where the interactions between particles depend on their relative positions [13,33-38].

Recently, the psychological status of the pedestrians per $s e$ is also taken into account in determining the dynamics of their movements with respect to time [39]. Generally, in most scenarios (such as in a hallway, or crossroad) the pedestrians like to move without bodily contact with other individuals, and are eager to arrive at their next destinations as soon as possible. That is, people would like to keep their own space and moving quickly with less disturbance of the rhythm of movement.

Anticipation is one of psychological mechanisms to guide the movement of the pedestrians with less bodily contact, i.e., when moving on the road, the pedestrians always tend 
to anticipate the others' positions and their moving tendencies to avoid any potential collisions in the near future. With the anticipation mechanism, the interaction between any pair of agents depends not only on their relative positions, but also on their velocity difference. A recent research work indicated that the estimation time for collision is a typical characteristic of the pedestrian dynamics [3].

In contrast to the extensive investigations of the mechanism of avoiding collision, the issue of quickly moving is little explored in the literature so far [40-42]. In this work, we try to fill this gap by taking into account both the factors of avoiding collision and quickly moving to study the collective behavior of pedestrians in three typical scenarios. Our principal idea is to consider the flow lanes (generated by the pedestrians just along the ahead or behind of their moving directions) as the object entities for interaction. We presume that in order to achieve fast moving with less collision the individuals have a tendency to follow the fast pedestrian flow lanes with the same moving direction and keep apart from the opposite ones. It is convenient to cast such effect into physical interaction rules, where the individuals are either attracted or repelled by the flow lanes generated by those surrounding pedestrians. By means of extensive computer simulations and thorough analysis, we intend to figure out some general characteristics of the collective behaviors of humans in typical pedestrian scenarios.

\section{THE MODEL}

As in common practice [12], we treat the pedestrians as active particles, whose motion with respect to time depend on serval types of forces.

The first one is the endogenous self-driving force. Since each particle $i$ has its own destination such that there exists a self-propelled force to guide its movement

$$
\vec{F}_{i}^{s p}=\beta\left(\vec{v}_{0, i}-\vec{v}_{i}\right)
$$

where the self-propelled force is aligned with the vector difference of pedestrian heading direction $\vec{v}_{i}$ and its desired velocity $\vec{v}_{i, 0}$, and its magnitude is proportional to the length of the vector difference. The prefactor $\beta$ characterizes how fast the particles react to the difference.

Second, we consider the repulsive force whenever two particles are too close to each other, which is defined as

$$
\vec{F}_{i}^{r p}=\alpha \sum_{j}\left(1-\frac{r_{i j}}{2 r_{0}}\right) \hat{r}_{i j}
$$

The repulsive force between particle $i$ and $j$ works when their physical distance satisfies $r_{i j}<2 r_{0}$, where $r_{0}$ is the radius of each particle (for simplicity, all the particles are identical). The quantity $\vec{r}_{i j}=\vec{r}_{i}-\vec{r}_{j}$ characterizes the relative displacement between them, where $r_{i j}$ is the length of $\vec{r}_{i j}$, and $\hat{r}_{i j}$ is the unit vector of $\vec{r}_{i j}$, and $\alpha$ is a proportional factor.

Third, we account for the interaction force induced by the anticipation mechanism. Whenever moving forward, the agents always detect the state of surrounding circumstance to anticipate the possibility of collisions and slow down their walking speed where necessary. Following Refs. [31,33-38], the calculation of the interaction force due to anticipation is
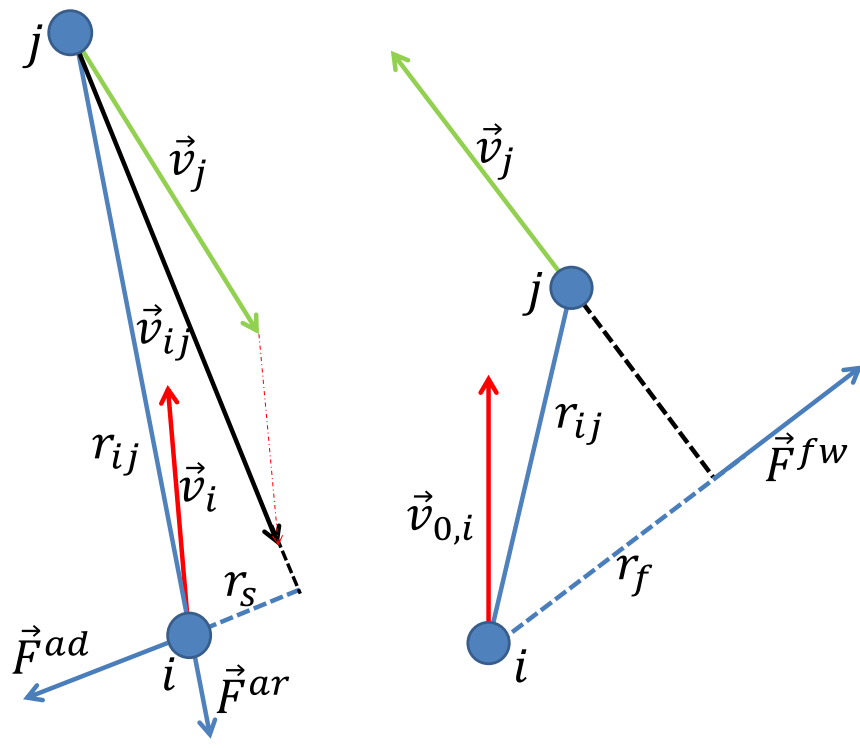

FIG. 1. Graphical illustration of our model. Whenever the angle between $\vec{v}_{i}$ and $\vec{v}_{j}$ is greater than $\pi / 2$ (left), collision might arise on the direction of relative movement $\vec{v}_{i j}=\vec{v}_{j}-\vec{v}_{i}$ provided that both the agents keep their moving trends unchanged. The agent $i$ anticipates such situation and will try to avoid collision by slow down its speed as if it is acted upon the repulsive force, exerted by the reverse flow lane along the direction $\vec{v}_{i j}$. If the included angle between $\vec{v}_{i}$ and $\vec{v}_{j}$ is less than $\pi / 2$ (right), the agent $i$ has a tendency to follow the trails of the "peer partner" $j$ trying to reach its destination quickly and with less collisions, as if it is attracted by the same direction flow lane generated by the agent $j$.

based on the anticipation distance, which is associated with the psychological attitude on the surrounding circumstance of the agents $[43,44]$. Generally, one person's psychological desired space is small when the surrounding crowd density is dense, which means that even they might be quite close to the others, they would accept the status and make slight (or no) response to the situation. By contrast, when the crowd density is sparse, even they could walk on smoothly without body contact with others, they would be unsatisfied to walk with strangers shoulder by shoulder. In light of these points, we take psychological desired distance with others as a relevant index to measure potential collision, and define the interaction force due to anticipation as

$$
\vec{F}_{i}^{a d}=\gamma\left(1-\frac{r_{s}}{d_{s}}\right) W^{a d}\left(\hat{v}_{i j} \times \hat{z}\right),
$$

where $d_{s}$ stands for the psychologically desired (or tolerant) distance, $r_{s}$ is the estimated distance of collision (whose value is determined by $r_{i j}\left|\sin \left(\theta_{v, r}\right)\right|$, where $\theta_{v, r}$ is the included angle between the vectors $\vec{r}_{i j}$ and $\vec{v}_{j i}$, see Fig. 1), and $\gamma$ is a prefactor. Note that the anticipative force is at work only when $r_{s}<d_{s}$. The quantity $W^{a d}=\frac{v_{i j}}{r_{i j}} \cos \left(\theta_{v, r}\right)$ characterizes the extent of anxiety of the agents for potential collisions, which turns into the driving force to adjust the rhythm of movement. Basically, $W^{\text {ad }}$ should be proportional to the magnitude of the relative velocity of the two agents $\hat{v}_{i j}$, the cosine of $\theta_{v, r}$, and be inversely proportional to distance of agent $i$ to the lane induced by $j$. For anticipative force, the direction of the unit 
TABLE I. A summary of the parameters used in our model.

\begin{tabular}{lcc}
\hline \hline$\alpha$ & fixed as 75 & $\begin{array}{c}\text { Characterizing the intensity of bodily repulsive interaction } \\
\beta\end{array}$ \\
$\gamma$ & fixed as 3 & $\begin{array}{c}\text { Reciprocal of the characteristic time for self-acceleration and self-deceleration } \\
\text { fixed as } 1\end{array}$ \\
& $\begin{array}{c}\text { Proportional coefficient for the repulsive or attractive forces exerted by the pedestrian flow lanes, } \\
\text { its magnitude is as the same order as } \beta \text { so that the former two types of forces are of the same } \\
\text { order as the self-propelled force }\end{array}$ \\
$r_{0}$ & fixed as $0.3(\mathrm{~m})$ & Radius of a typical pedestrian based on empirical observation \\
$\bar{v}_{0}$ & fixed as $1.34(\mathrm{~m} / \mathrm{s})$ & Average walking speed of the pedestrians \\
$d_{m}$ & chosen as $2,5,10(\mathrm{~m})$ & Maximum range of visibility of the pedestrians (lower for denser systems, see Appendix) \\
$d_{s}$ & free parameter & Characterizing the interaction range of the repulsive force due to the anticipation mechanism \\
$d_{f}$ & free parameter & Characterizing the interaction range of the attractive force due to the attraction mechanism \\
\hline \hline
\end{tabular}

vector $\hat{z}$ is defined as $\hat{z}=\left(\vec{r}_{i j} \times \vec{v}_{i j}\right) /\left|\vec{r}_{i j} \times \vec{v}_{i j}\right|$ such that the dodging direction is always perpendicular to and away from the reverse flow lane, denoted by $\vec{F}^{a d}$ in the left panel in Fig. 1 . In any case the included angle between $\vec{r}_{i j}$ and $\vec{v}_{i j}$ is zero, $\hat{z}$ will be a unit vector perpendicular to the plane, inward direction. In this way, the agents follow a right-hand traffic rule for frontal approaches $(\theta=0)$.

In case the estimated distance of collision $r_{s}$ is less than $2 r_{0}$ indicating the adjustment of the direction of velocity, i.e., Eq. (3), may not work for avoiding the occurrence of a collision, we assume the presence of another repulsive force to drive the agent's motion

$$
\vec{F}_{i}^{a r}=\gamma\left(1-\frac{r_{s}}{d_{s}}\right) W^{a d} \hat{r}_{i j},
$$

whose magnitude is exactly the same as in Eq. (3), but with a different direction.

Finally, we also consider the attractive effect of the pedestrian flow lanes, as indicated in the right panel of Fig. 1. The rationale is that, besides the tendency to keep away from the reverse flow lanes, each agent is willing to follow those same direction flow lanes (i.e., flow lanes pointing to the same target direction) trying to reach the destination as efficiently as possible. (Here we remind that only those pedestrian flow lanes with potential crossover with the forward path of the focal agent will exert either an attractive or a repulsive force on it.) We define the attractive force on the agent $i$ exerted by the same direction flow lane from the agent $j$ as

$$
\vec{F}_{i}^{f w}=\left\{\begin{array}{cl}
\gamma \quad\left(\frac{r_{f}}{d_{f}}\right)^{2} W^{f w}\left(\hat{v}_{j} \times \hat{z}\right), & \text { if } r_{f}<d_{f} \\
0, & \text { otherwise }
\end{array},\right.
$$

where $r_{f}=r_{i j}\left|\sin \left(\theta_{v j, r}\right)\right|$ representing the vertical distance from the position of $i$ to the black dash line (the flow lane generated by the individual $j$ ), $\theta_{v j, r}$ is the included angle between the vectors $\vec{r}_{i j}$ and $\vec{v}_{j}$, and $d_{f}$ depicts the maximum distance that an agent will start to follow (i.e., to be attracted by) the lanes of the others. The quantity $W^{f w}=\frac{v_{j}}{r_{i j}} \hat{v}_{j} \cdot \hat{v}_{0, i}$ measures the extent of attraction of the flow lane. Obviously, a larger speed and more similar direction of movement will exert greater attraction to the focal agent $i$. Similarly, for attractive force, $\hat{z}=\left(\vec{v}_{j} \times \vec{r}_{i j}\right) /\left|\vec{r}_{i j} \times \vec{v}_{j}\right|$ so that the cross product of $\hat{z}$ and $\hat{v}_{j}$ is pointing vertically (and closing) to the pedestrian flow lane, denoted by $\vec{F}^{f w}$ in the right panel in Fig. 1 .

Taken together, in our model the movement of pedestrians are mainly driven by self-propelled force, physical body collision, and the repulsive or attractive forces exerted by the surrounding pedestrian flow lanes. Self-propelled force and physical body collision are commonly considered in the literature to study the behavior of pedestrians, while the repulsion or attraction due to the pedestrian flow lanes are newly introduced ingredients in our current model. We remark that the interaction forces exerted by the pedestrian flow lanes are heuristically inspired in terms of the psychological status of the pedestrians: the tendency of keeping away from the reverse flow lanes reflects the instinctive reaction of the pedestrians to avoid potential collisions, and the trend of following the flow lanes pointing to the same targeted direction implies their eagerness for quickly moving.

Both mechanisms of avoiding potential collisions and seeking for quickly moving will be only at work provided that the distance between the considered pair of agents is less than the average maximum range of visibility $d_{m}$, see Appendix for a detailed estimation of its value. To reduce the complexity of our model, the parameters $\alpha, \beta, \gamma, r_{0}, \bar{v}_{0}$, and $d_{m}$ are chosen as fixed values in terms of either empirical observations or theoretical estimations, while the only two free parameters are $d_{s}$ and $d_{f}$, characterizing the interaction range of the repulsive and attractive force exerted by the pedestrian flow lanes, respectively. In particular, we choose $d_{m}=10,5$, and 2, respectively, for sparse, moderate and dense pedestrians studied below. Unless otherwise specified, we use $\alpha=75,{ }^{1} \beta=3$, $\gamma=1$, and $r_{0}=0.3$ for simulations in this work, and set the expected speed distribution as a Gaussian distribution with mean value $\bar{v}_{0}=1.34$ and standard deviation 0.26 . A summary of the parameters is listed in Table I.

\section{SIMULATION RESULTS AND ANALYSIS}

The two crucial parameters in our model are the psychologically desired distance $d_{s}$ away from others and the maximum distance $d_{f}$ of attraction by the same direction flow lanes. In what follows, we present and analyze the simulation results of our model by tuning these two parameters for several typical pedestrian scenarios, such as pedestrians in a straight corridor with bidirectional flows, pedestrians in a square with crossing

\footnotetext{
${ }^{1}$ Note that we have adopted a sufficiently large $\alpha$ so that the unphysical phenomenon of penetration, i.e., two pedestrians approaching centrally may penetrate each other when their relative speed is large enough in terms of Eqs. (1)-(5), will never occur.
} 
Initial waiting area Observing area Initial waiting area

3

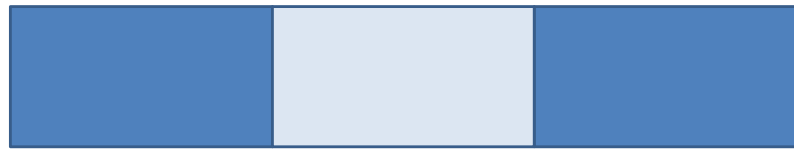

20

20

FIG. 2. Schematic illustration of our simulation setting for the bidirectional pedestrian flows.

flows, and panic pedestrians swarming to a narrow gate in the case of evacuation dynamics. These classical scenarios have been extensively studied in the literature but with different microscopic interaction rules among the agents [3,21].

\section{A. Bidirectional pedestrian flows in a straight corridor}

First, we consider the corridor case where the agents are separated into two groups walking on with opposite destinations. For simplicity, we assume that the number of individuals in the two groups are equal and all the agents stay at the waiting area shown in Fig. 2 initially. Then the agents begin to move according to the rules Eqs. (1)-(5). We make statistical analysis of the moving behavior of the pedestrians in the middle observation area. The width of the corridor is set to 3 , which is of 10 times the radius of an individual, suggesting that it allows at most 6 individuals to pass through shoulder by shoulder. We vary the number of agents from 100 to 300 for doing statistics of the system order parameter in the observation area.

In a straight corridor, people are generally so concerned with the oncoming individuals. Thus it is reasonable to assume a large anticipation distance in such situation. In particular, we adopt $d_{s}=1.5$ so that all the agents have a strong tendency to avoid collision. By varying $d_{f}$ and $N$ we observe some interesting emergent collective motion patterns, which are presented in Fig. 3. For sparse systems, people tend to walk smoothly and the microscopic movement behavior is independent of $d_{f}$, whereas in the case of dense systems, large $d_{f}$ results in the lane separation, which guarantees a high efficiency of passing through, while small $d_{f}$ leads to the arise of jamming.

To specify the effect of $d_{f}$ on the bidirectional flow movement, we measure some physical quantities illustrated below. First, we define the local order parameter of the particle $i$ by the mean of projection of the others' velocity on $i$ 's in the rectangle shown in Fig. 4.

$$
o_{i}=\frac{1}{N_{C_{i}}} \sum_{j \in C_{i}} \hat{v}_{i} \cdot \hat{v}_{j},
$$

where $N_{C_{i}}$ is the number of particles in the rectangle region. By averaging this quantity over all the agents, we obtain the average local order parameter of the system at each moment $l$ as

$$
O_{l}=\frac{1}{N} \sum_{i} o_{i},
$$

where $N$ is the system size. (a)

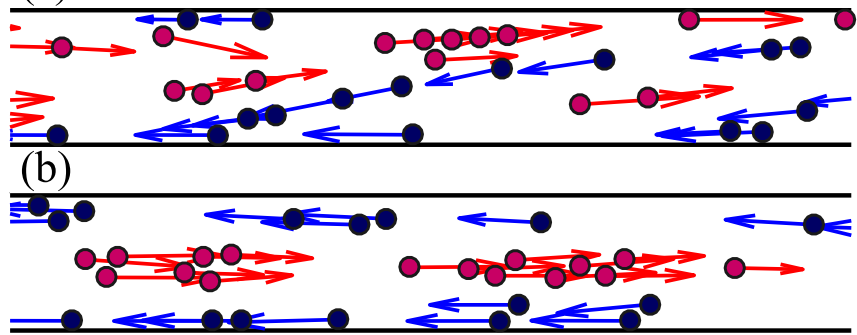

$\overline{(c)}$

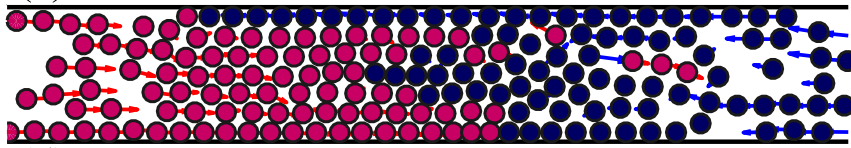

(d)

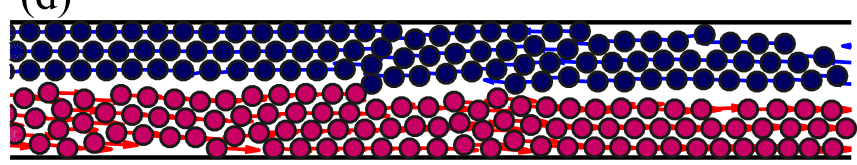

FIG. 3. Typical snapshots of the pedestrians in the corridor case for different combinations of $N$ and $d_{f}$. The parameter $d_{s}$ is fixed as $1.5, d_{f}=0.1$ in (a) and (c), and $d_{f}=1$ in (b) and (d). In sparse systems (a) and (b), $N=50$, the agents can pass through efficiently, while in dense systems (c) and (d), $N=300$, only with the presence of a strong attraction effect does the separation of global flow lanes emerge.

The time sequences of $O_{l}$ are plotted in Fig. 5, which allows us to figure out the process of lane formation. According to our simulation procedure, $O_{l}$ is initially high since all the agents are staying at the boundaries of the observation area. When the marching fronts of the two groups are getting closer, the average order parameter begins to drop, see Fig. 5. Nonetheless, we observe that a large range $d_{f}$ of attraction by the same direction flow lanes helps to the formation of collectively moving clusters (usually resulting in a large $O_{l}$ ), no matter what the system size $N$ and the psychologically desired distance $d_{s}$ are. We also find that a dense system with greater $d_{f}$ can keep lanes more stably [the flat curves for large $d_{f}$ in Figs. 5(c) and 5(d), and also see Fig. 3(d)], because once the global flow lanes emerge, those nearby agents have to follow the pattern formatted, since any disobedience will lead to severe collisions with the agents in the reverse flow. Whereas in sparse systems, even just with the avoidance mechanism, people can pass through efficiently, similar to the results in [3]. We point out that the formatted flow patterns are vulnerable without the support of a large number of agents, where they are likely to merge and split for a small system of $N=100$.

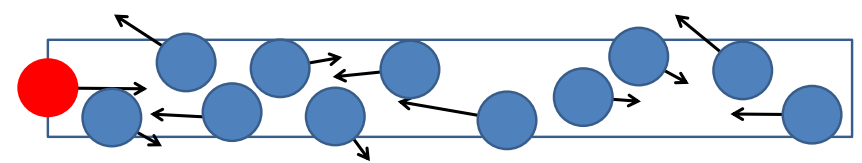

FIG. 4. Sketch of the calculation of the local order parameter. For each agent $i$ (the red one), we select a rectangular area (width is $4 r_{0}$ and length is 10) along the direction of its destination (here the right) and measure the averaged velocity projection of the others in the area in the direction of $\vec{v}_{i}$. 

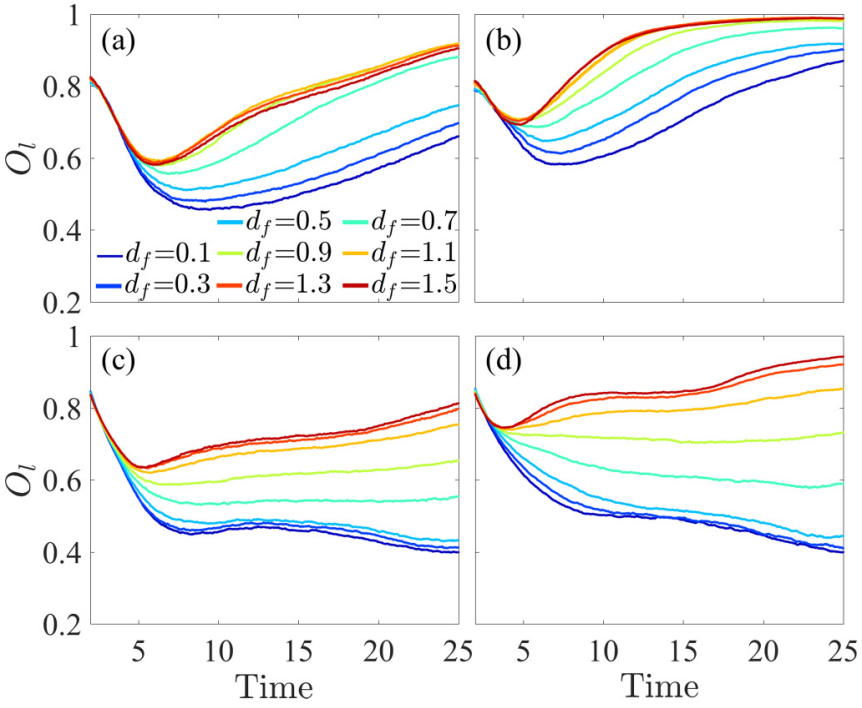

FIG. 5. Time series of the mean local order parameter $O_{l}$ with different combinations of the parameters $N, d_{s}$ and $d_{f} . N=100$, $d_{s}=0.6$ in (a), $N=100, d_{s}=1.5$ in (b), $N=300, d_{s}=0.6$ in (c), and $N=300, d_{s}=1.5$ in (d).

We can find some useful clues on the pedestrian traffic jam from Figs. 3(c) and 3(d) for dense systems. For small $d_{f}$, the agents are weakly influenced by their neighbors moving on the same direction, and when the front agents of the two groups are contacting, they are easy to form a confrontation at the junction. The oppositely marching people without compromise further duce the available width of the road, and finally leading to the emergence of jamming, resulting in low values of the ordered parameter $O_{l}$, shown in Figs. 5(c) and 5(d). In Ref. [45], both bidirectional and unidirectional pedestrian flow have been investigated, and it was found that the clogging occurs in local area and expand gradually until the agents overoccupy the load. However, for a sufficiently large $d_{f}$, the great attraction forces exerted by those peer partners can effectively prevent the formation of confrontation [even if a deadlock is occasionally formed at the junction, a tiny fluctuation (noise effect) would likely lead to the gathering of agents with the same destination at one side of the corridor], which in turn gives rise to the formation of flow patterns observed in Fig. 3(d). We point out that a similar result is found in Ref. [46], where globally ordered pedestrian flow lanes are formed simply due to the symmetry breaking.

Another statistical quantity we measured is the mean selfprojection of velocity, which is defined as the magnitude of $\vec{v}_{i}$ projected into the direction of $\hat{v}_{0, i}$

$$
v_{p, i}=\vec{v}_{i} \cdot \hat{v}_{0, i} .
$$

The mean self-velocity projection $\left\langle v_{p}\right\rangle=\sum_{i} v_{p, i} / N$ characterizes to what extent the agents are walking in their desired directions. If this quantity is equal to the mean expected speed (which is set to 1.34 in our current study), then all the agents just walk with the most efficient way to their destinations. In Fig. 6, we plot $\left\langle v_{p}\right\rangle$ as a function of time for some combinations of the parameters. We find that compared with $O_{l}$, there is no evident decline for $\left\langle v_{p}\right\rangle$ for sparse systems, where the pedestrians may not walk in line or in lanes, but they can
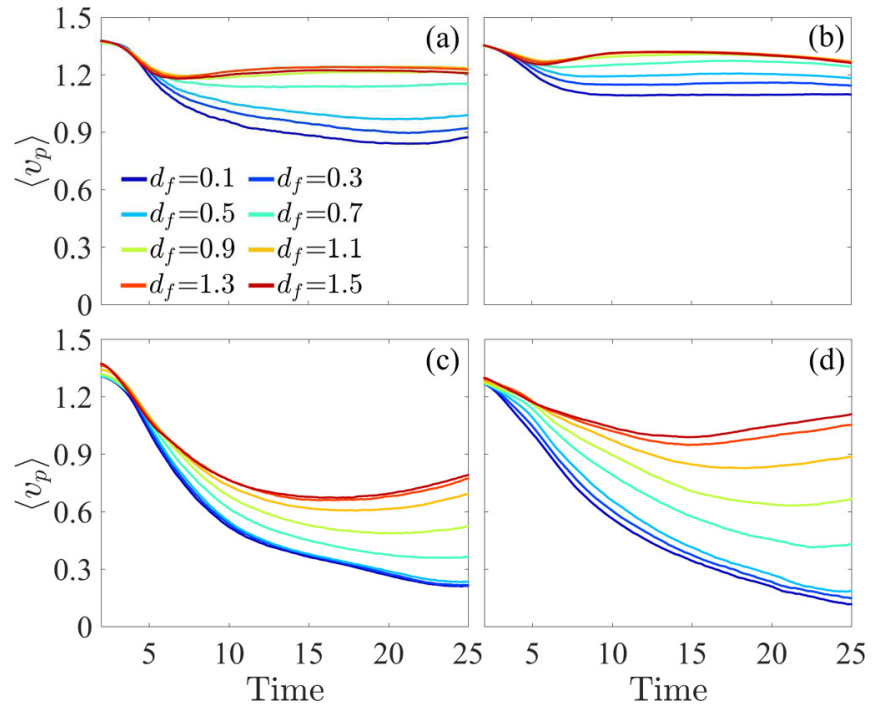

FIG. 6. Time series of the mean self-velocity projection $\left\langle v_{p}\right\rangle$ with different combinations of the parameters $N, d_{s}$ and $d_{f} . N=100$, $d_{s}=0.6$ in (a), $N=100, d_{s}=1.5$ in (b), $N=300, d_{s}=0.6$ in (c), and $N=300, d_{s}=1.5$ in (d).

keep high speed, owning to less collision probability in sparse crowd. For larger $d_{s}$ values, the agents are able to anticipate potential collisions with others earlier such that they can always walk on smoothly. As a consequence, large anticipation distance $d_{s}$ helps to improve the passing efficiency no matter what the system size is.

The arise of jamming in dense systems with low $d_{f}$ is also reflected by the behavior of $\left\langle v_{p}\right\rangle$, as shown in Figs. 6(c) and 6(d). As time goes on, more and more agents swarm into the observation region, and are hindered there for small $d_{f}$, and their passing speeds in the observation area declines rapidly. The crisis in this situation is that once a jam is formed, it is hardly vanished via self-organization of the agents. Broken of self-organization means less order and more blocking units slowing down the passing flow of the pedestrians. In contrast, higher $d_{f}$ helps agents with the same destination to form clusters with separate lanes, and once such lanes have emerged, $\left\langle v_{p}\right\rangle$ increases continuously with respect to time. We remark that our current findings are consistent with those from real experiments in Ref. [8] in studying the process of flow lane formation.

In another experimental study of bidirectional pedestrians in a straight corridor [18] with length 12 and width 20, it was found that the main behavior of avoidance is that people can steer their walking direction frequently. We yield the same result in our theoretical model, where avoidance of potential collisions and attraction by quickly moving lanes are the two mechanisms to reduce the probability of collision. We emphasis that mutual avoidance is the dominating rule among sparse pedestrians, but in dense systems avoidance behavior becomes less efficient, while following fast moving peer partners will serve as a vital role in driving the agents to form stable flow lanes.

To check the robustness of the above results, we further study the situation where the pedestrians are initially distributed randomly in a square area, whose top and bottom 
(a)

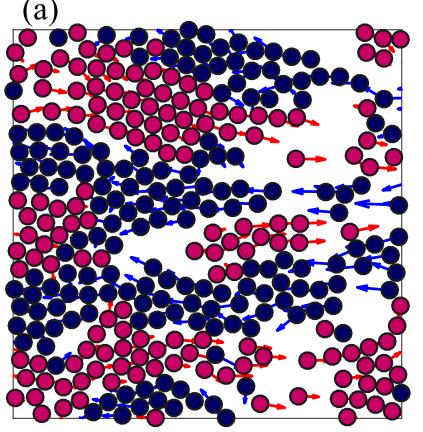

(b)

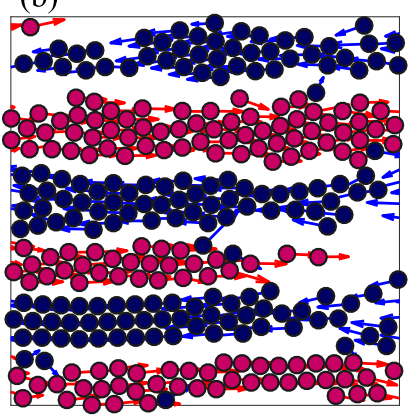

FIG. 7. Typical snapshots of the bidirectional pedestrian flows in the stationary state in a square area, where the agents are reinjected to the system whenever they are leaving out at the boundaries. The observation region for this demonstration is a square with linear length 12 , the number of agents in both simulations is $N=400$ and $d_{f}=0.1,1.0$ respectively in (a) and (b). Stable pedestrian flow lanes emerge in the case of large attraction distance $d_{f}$, right panel.

boundaries are of periodic boundary condition. The pedestrians move either from left to right or vice versa. To conserve the total amount of the pedestrians, if an agent reaches its target at left or right side, we will inject a new one into the system from the opposite side with distance 2.0 to the boundary, and its $y$-axis position is randomly chosen.

We plot the typical stationary results in Fig. 7, where the formation of visible flow lanes can be observed. Once again, for large values of $d_{f}$, the flow lanes are more distinguished with stable structures.

To better understand the simulation results, we measure the velocity-direction correlation on the vertical distance, which can be used as a rough estimation of the width of the formatted flow lanes. The results are summarized in Fig. 8. Although the amplitude of the correlation function decays with vertical dis-
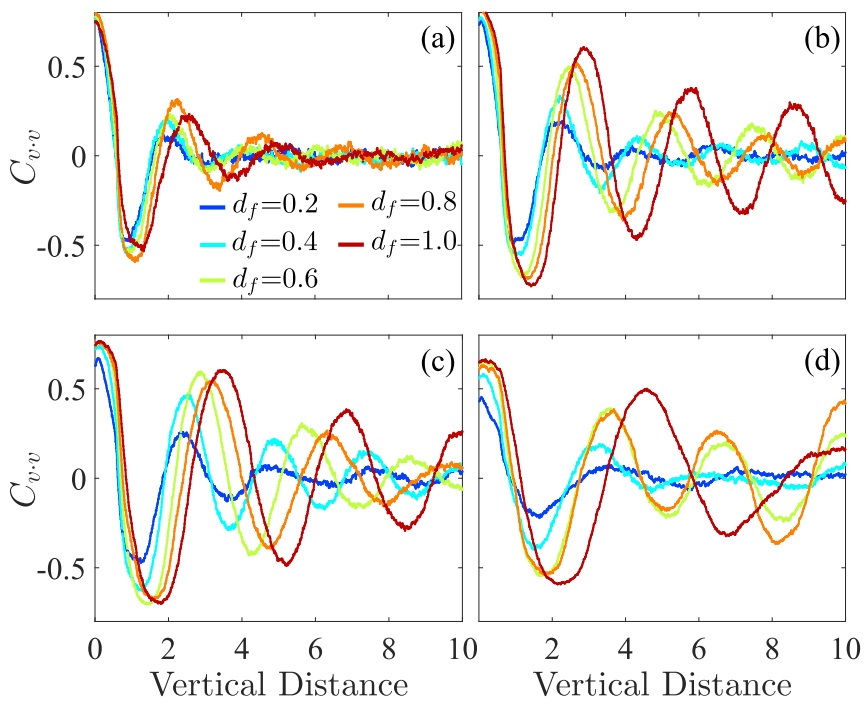

FIG. 8. Average velocity-direction correlation $C_{v \cdot v}$ of the agents as a function of the vertical distance for different $N$ and $d_{f}$, which is measured as $C_{v \cdot v}(\Delta y)=\left\langle C_{v \cdot v}^{i j}\left(\left|y_{i}-y_{j}\right|\right)\right\rangle$, if $i \neq j$, and $C_{v \cdot v}^{i j}\left(\mid y_{i}-\right.$ $\left.y_{j} \mid\right)=\hat{v}_{i} \cdot \hat{v}_{j}, \Delta y=\left|y_{i}-y_{j}\right| . N=100,200,400$, and 800 respectively in (a), (b), (c), and (d).

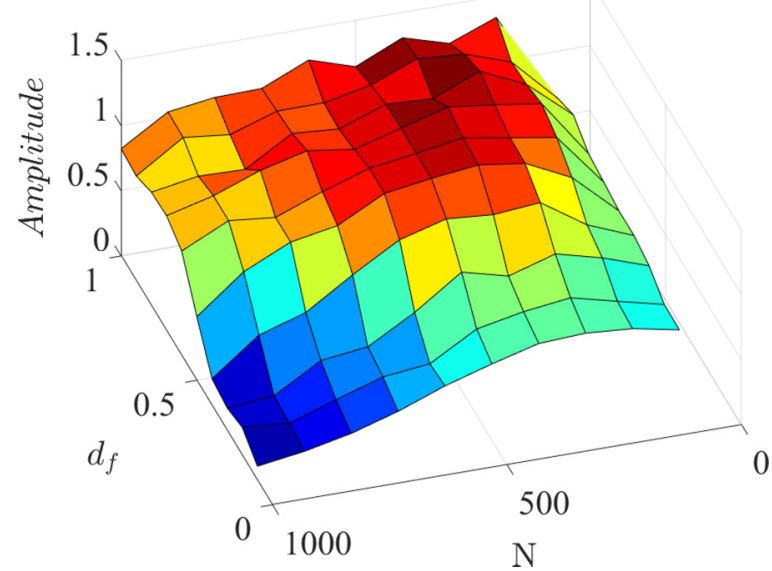

FIG. 9. Relative amplitude of the velocity-direction correlation function as a function of the parameters $N$ and $d_{f}$, and $d s$ for these simulations is fixed as 1.0.

tance, the period-like waveform indicates that the flow lanes with opposite directions occur alternatively and evenly, and its wave length represents approximately the width of the lanes. Particularly, the amplitude can signify how stable those lanes are: if the formed lanes rarely change over time, we would obtain relatively high values of correlation, otherwise the rapid changes of lanes would mask the correlation and result in vanishingly small amplitude. Therefore we can infer from Fig. 8 that in sparse systems, pedestrian flow lanes always change frequently no matter what $d_{f}$ is. With the increase of density, however, large $d_{f}$ will result in more stable and wider lanes markedly. This point is easily understandable, a wider attraction distance will drive more peer partners to follow, which benefits the formation of wider flow lanes, and with agents swarming into these lanes continuously, the newly joined pedestrians will further make the lanes even more stable.

In Fig. 9, we calculate the relative amplitude of the correlation function by the second peak value subtracting the first trough value in Fig. 8 for different $N$ and $d_{f}$. The relative amplitude can be regarded as the degree of purity of the adjacent lanes, which may also serve as another indicator of the order of the system. Obviously, in Fig. 9, we witness that $d_{f}$ plays a vital role in dense system with $N \in(200-600)$ [the density $\rho \in(0.5-1.5)]$, and larger $d_{f}$ brings more neat lanes.

\section{B. Crossing pedestrian flows in a crossroad}

Now we turn our attention to the crossing motion scenario of two groups of pedestrians with mutual orthogonal target directions (for convenience, we call them as group one and group two, respectively). As before, we first consider the situation of no reentrance of the agents whenever they are leaving out the simulation area, see Fig. 10. Initially, the two groups with mutual orthogonal destinations are placed in the waiting area, and then they are allowed to move simultaneously. When they enter the crossing area, we make some statistical measurements on their moving behavior. In a previous experiment [17], it was suggested that people may underestimate the risk of collision when they just notice each other, and the 


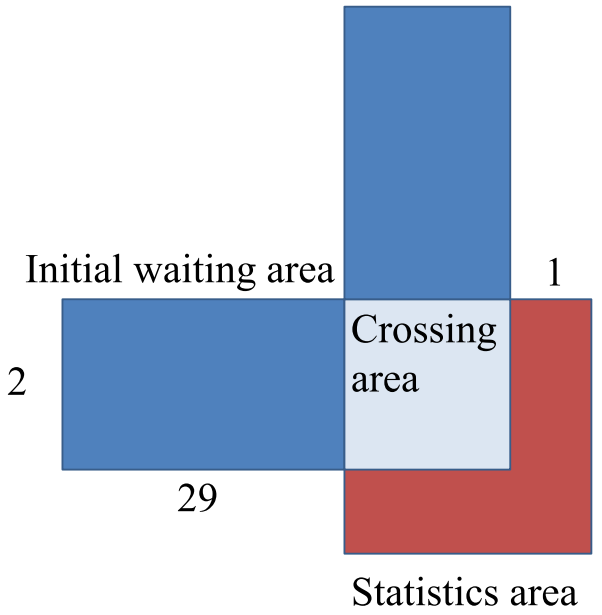

FIG. 10. Sketch for the crossing pedestrian flow simulation. Initially, the agents are standing in the waiting areas (blue zone), whose length is 29 and width is 2 . We measure their moving behavior when they are entering the crossing area (light blue zone) and the statistics area (red region).

estimation of collision risk is increased as they are getting closer. Our aim here is to investigate how the anticipation distance $d_{s}$ influences the agents to avoid each other.

In Fig. 11, we present the typical snapshots for the crossing flow scenario for two combinations of $d_{s}$ and $d_{f}$. When the agents are of strong tendency to avoid collision $\left(d_{s}=1.5\right)$ but of weak inclination for following fast moving peer partners $\left(d_{f}=0.1\right)$, we observe interesting phenomena, where the agents with different targets are capable of organizing themselves into blocks. when one block of agents start to pass the crossing area as a whole, the agents from the other group will stay waiting, and then the reverse case happens. By contrast, for the system with $d_{s}=0.6$ and $d_{f}=1.5$ (agents take care less for collision, but serious for quickly moving), the agents fall into a state of disarray and no one can pass cross the other-side crowd to reach the destination efficiently.

To get a quantitative view, we check the proportion $p(t)$ of agents from one certain group having entered the red color region in Fig. 10 as a function of time. To be more specific, if there are $a$ agents from the group one and $b$ agents from
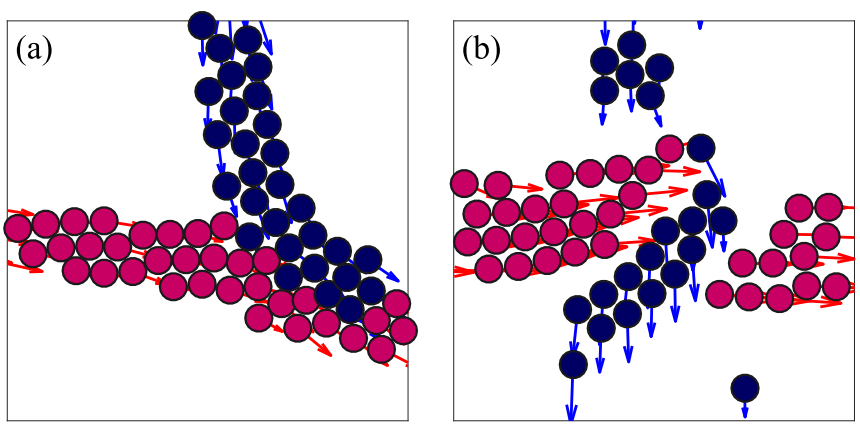

FIG. 11. Typical snapshots of the crossing pedestrian flows for two different combinations of $d_{f}$ and $d_{s}, d_{s}=0.6, d_{f}=1.5$ in (a) and $d_{s}=1.5, d_{f}=0.1$ in (b). The number of agents in both simulations is $N=200$.
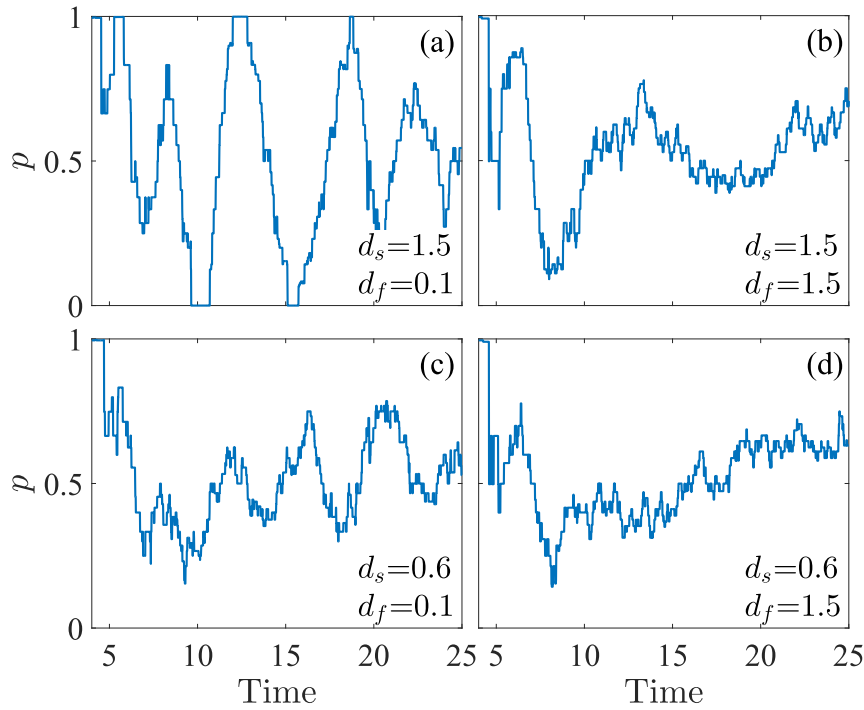

FIG. 12. Time series of the proportion $p(t)$ of agents from one certain group having entered the red color region in Fig. 10. The results are for one single simulation realization with no reentrance of the agents whenever they are leaving out the simulation area.

the group two have appeared in the red region at time $t$, then $p(t)=a /(a+b)$. The time series of $p(t)$ is plotted in Fig. 12, which can tell us at a certain moment which direction (horizontal or vertical) is the major one with active pedestrian flow, i.e., almost only the agents along this direction are moving, while those along the other one are waiting. From Fig. 12, we observe rhythmic overturn of $p(t)$ representing the arise of alternate passing and waiting phenomenon. On the contrary, irregular fluctuation of $p(t)$ around 0.5 just means, without mutual compromise as shown in Fig. 11(a), people get into disordered motion.

In order to get a deep insight, we calculate the quantity

$$
\langle p\rangle=\int_{0}^{T}|p(t)-0.5| d t / T
$$

during a long time interval $T$, whose magnitude thereby specifies whether the phenomenon of alternate waiting and passing is significant or not. The greater $\langle p\rangle$ is, the more striking of the phenomenon. The ensemble average results of $\langle p\rangle$ for different system size and parameters $d_{f}$ and $d_{s}$ are summarized in Fig. 13. In sparse systems, the moving pattern of the pedestrians seems independent of $d_{f}$ and $d_{s}$, which is understandable since great fluctuations of small systems will erase the statistical meaning of our measurements. For denser systems with large $d_{s}$, the appearance of a large number of agents in one group will force the front individuals of the other group decelerating their speeds in front of the crossing area so that they tend to aggregate there until they pass together in due course. Our simulation results are in well agreement with the experimental studies for mutual crossing scenario [18], where alternate waiting phenomena are frequently occurred.

A counterintuitive phenomenon is the arise of disordered moving pattern in dense systems with large $d_{f}$, which indicates that following fast flow lanes does not always help to the formation of highly ordered motion, see Figs. 13(c) and 13(d). In Fig. 11, we find that the attraction effect by the same 

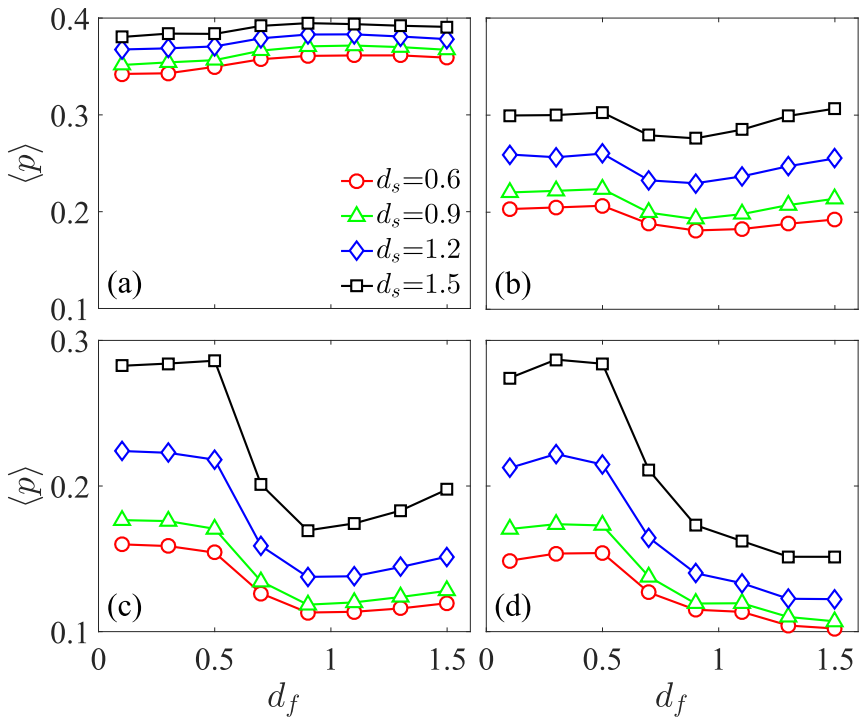

FIG. 13. The quantity $\langle p\rangle=\int_{0}^{T}|p(t)-0.5| d t / T$ as a function of the attraction distance $d_{f}$ for different system size $N$ and anticipation distance $d_{s}, N=50,100,150$, and 200 in (a), (b), (c), and (d). The larger value of $\langle p\rangle$, the more striking of the alternate waiting and passing phenomenon.

direction pedestrian flow lanes has pushed people into narrow lines for large $d_{f}$, hence much part of the road are wasting. For the crossing pedestrian flows, the width of the channel affects greatly the efficiency of passing. If the agents were evenly dispersed on the road, the pedestrian density in the crossing area would become lower so that individuals could be easier to avoid the others instead of facing collision of two screwing long queues.

The mean self velocity projection $\left\langle v_{p}\right\rangle$ in the crossing pedestrian flow scenario is shown in Fig. 14. In sparse systems, the collective motion behavior of the pedestrians is

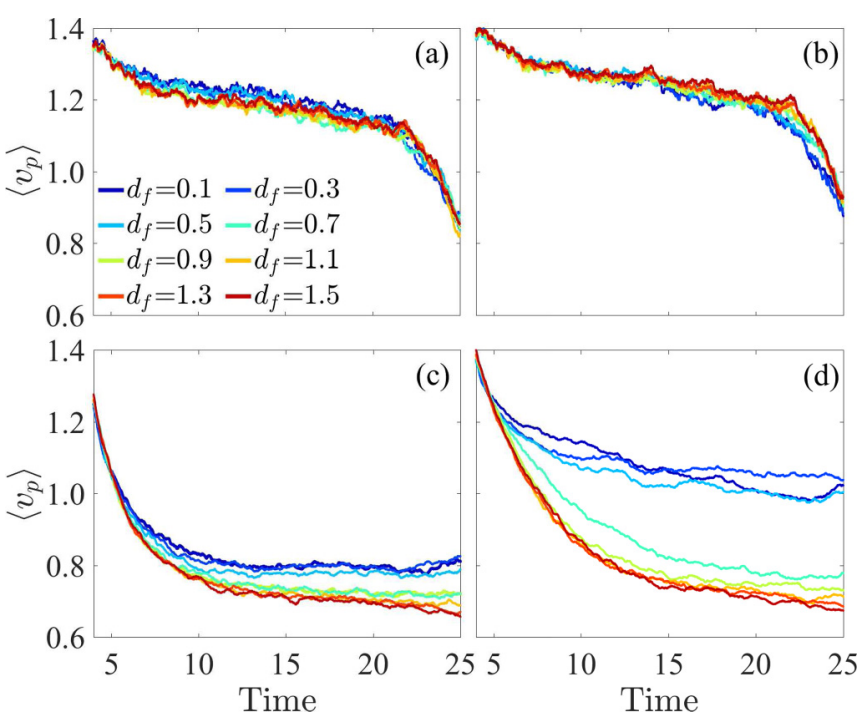

FIG. 14. Time series of $\left\langle v_{p}\right\rangle$ of the agents in the crossing area for different combinations of the parameters $N, d_{s}$ and $d_{f}, N=50$, $d_{s}=0.6$ for (a), $N=50, d_{s}=1.5$ for (b), $N=200, d_{s}=0.6$ for (c), and $N=200, d_{s}=1.5$ for $(\mathrm{d})$.
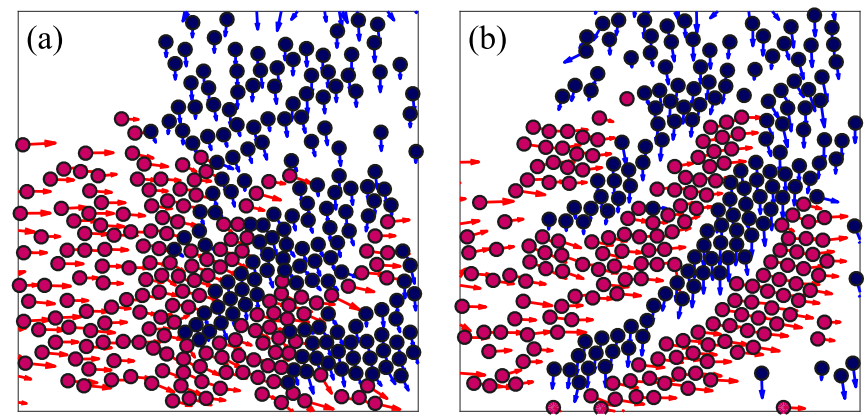

FIG. 15. Typical snapshots of the crossing pedestrian flows where the agents are allowed to rejoin into the systems whenever they are leaving out from the right or bottom boundaries. The parameter $d_{f}=0.1$ and the number of agents is $N=400$ in both simulations. The parameter $d_{s}=0.6$ in (a) and $d_{s}=1.5$ in (b)

insensitive to both the parameters $d_{s}$ and $d_{f}$, see Figs. 14(a) and 14(b), whereas in dense systems, the anticipation distance $d_{s}$ plays a dominating role in determining the passing efficiency of the pedestrians. For small $d_{s}$, the performance of the agents is poor, shown in Fig. 14(c). Whenever the agents anticipate potential collisions very early (large $d_{s}$ ), they are able to pass the crossroad quickly provided that the attraction effect among peer partners is weak (small $d_{s}$ ), see Fig. 14(d). Nevertheless, if the attraction effect is strong (large $d_{f}$ ), the passing efficiency is greatly inhibited again.

What about the collective motion pattern in the situation that the agents are allowed to rejoin the system whenever they leave out through the right or bottom boundaries? Interestingly, we find the emergence of skewed flow lanes for large anticipation distance $d_{s}=1.5$, shown in Fig. 15, which is quite different from the bidirectional pedestrian flows in a straight corridor. The skewed flow lanes appear alternately for the two groups with different targets such that it looks like the formatted lanes are moving themselves. By aid of such lane-moving mode, the agents hardly collide to the others, and the well-regulated lanes ensure that the agents walk efficiently toward their targets, since when they move forward, the others blocking in the front have also moved away at same time so that individuals in this situation seldom slow down their speed. Remarkably, we notice that with such skewed lane mode, the pedestrians try to bypass the others ahead from their back, which is in accordance with our daily experience that people usually prefer a early moving with slightly longer route rather than stop and wait for the pedestrians in front to pass. We remark that in a recent research [3], similar phenomenon is obtained with different microscopic interaction rules among the agents.

\section{Crowd evacuation in a room}

To check whether our current model is able to depict versatile collective motion of pedestrians, as a final attempt we study how the agents swarm quickly to narrow or bottleneck exit in the case of an indoor fire panic. The evacuation dynamics has been extensively investigated in the last decades [30,47]. Plenty of previous works have focused on the influence of the population density, door size, flow rate 

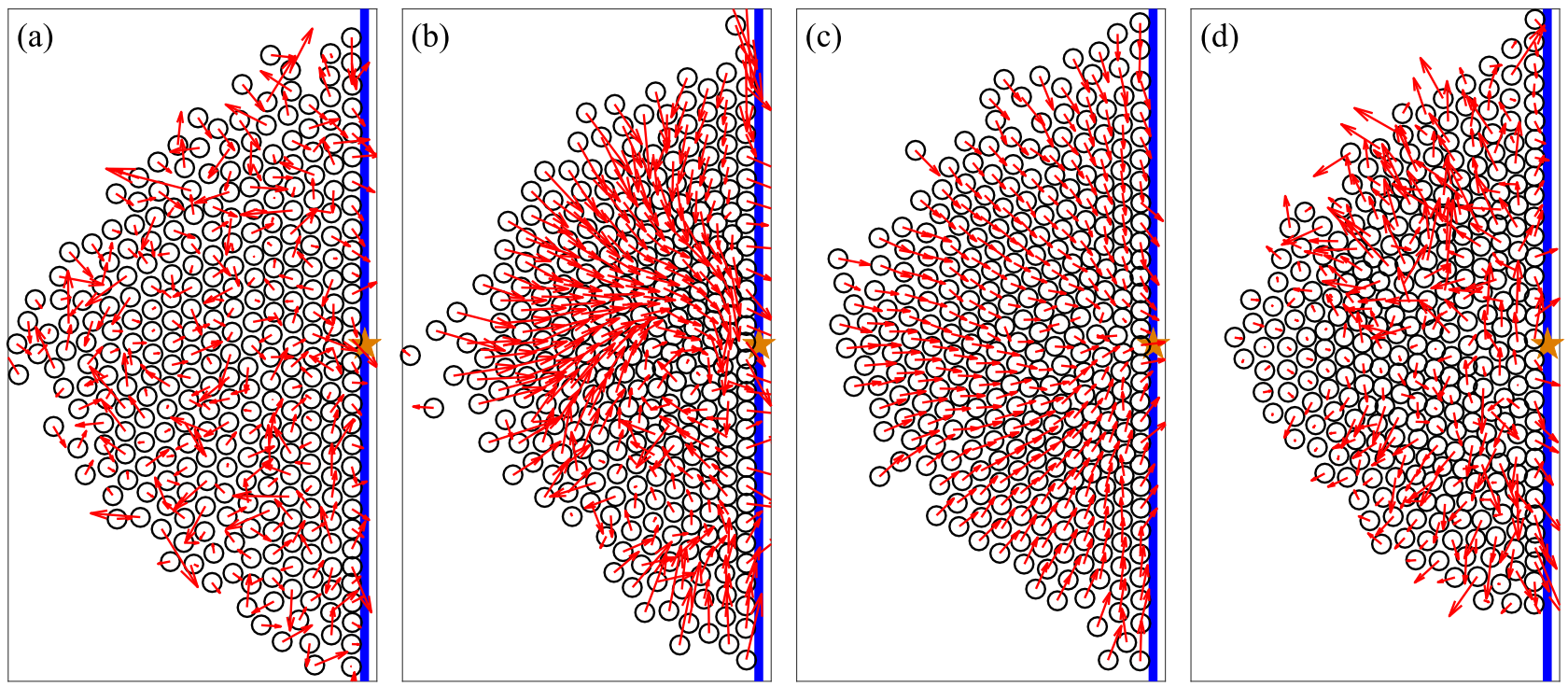

FIG. 16. Typical snapshots of the crowd evacuation in a room for different $d_{f}=0.1,1.2,1.5$, and 1.5 in (a), (b), (c), and (d). The other parameters are $N=300$ and $d_{s}=0.6$. In the case of evacuation, the agents have strong desire for moving to the exit point which marked as a star. For simplicity, however, we don't allow them to cross the wall, since the exit serves as a bottleneck extremely inhibiting the passing efficiency, especially in panic situations.

on the efficiency of evacuation and safety [31,43]. In a recent work [48], Bottinelli et al. investigated exclusively the stable patterns of the pedestrians gathering at the exit.

Following the research line of [48], we here intend to study the gathering patterns of the pedestrians near the exit entry. To do this, we make minor modifications of our model for the bidirectional and crossing pedestrian flows. A lot of existing work has suggested that in the panic situation, the behavior of human becomes irrational or even crazy [30,47]. Thus we assume that under such situation, the agents prefer mainly those high speed flow lanes without the consideration of whether the followed flows are really helpful to close to the exit. For this reason, we change the function $W^{f w}$ to $W^{f w}=\frac{v_{j}}{r_{i j}}$ so that only the magnitude of the speed matters much, not the direction of the velocity. Since the desired direction of each agent is the vector from its location pointing to the exit, we regard that, apart from the four types of forces mentioned above, the exit point serves as a central force field exerting attraction force on all the agents. In particular, the central force exerted on agent $i$ is formulated as

$$
\vec{F}_{i}^{e x}=\omega\left(0-\vec{r}_{i}\right)=-\omega d_{\mathrm{exit}} \hat{r}_{i}
$$

where $\omega$ is fixed as $0.2, d_{\text {exit }}$ is the distance between the position of the agent $i$ and the exit. Note that we have selected the exit as the origin of the coordinates.

Figure 16 shows some typical snapshots of the agents for a system with $N=300$ and several $d_{f}$ (in all cases, $d_{s}$ is fixed as a small value 0.6 since in such crowding scenario the agents account mainly for quickly moving with less consideration of avoidance of collision). The agents are gathering to the exit and arranged into a semicircular arch shape [49]. Notably, we observe the endless process of swing, gathering, puffing, swing of the agents (typical videos are supplemented in Ref. [50])
We plot in Fig. 17 the spatiotemporal patterns of the average local density $\rho=\sum \rho_{i} / N^{2}$ and also those of the velocity projection along the direction pointing to the exit (e.g., in the direction of $-\hat{r}_{i}$ for the agent $i$ ) for several values of $d_{f}$. Interestingly, we find quasi-periodic motion behaviors of the agents. In the initial stage, all the agents are gathering to the exit point, driven by both the self-propulsion and the central force field. Accompanied by the aggregation process is the increasing of internal pressure in the crowd, and once the internal pressure is elicited to a sufficiently high level, repulsion interactions will induce inflation, suddenly pushing the agents from the inner area to the periphery. After that, the average local density in the room is decreased so that the attraction forces on the agents can surpass the repulsive ones again, and the individuals move to the exit subsequently, thereby the next round of gathering begins.

For large $d_{f}$, we find that the process of contraction and inflation happen in all the directions almost simultaneously, as shown in Figs. 16(c) and 16(d). Interestingly, when the range of attraction $d_{f}$ is decreased to appropriate level, say $d_{f} \approx 1.1$, we may obtain the anisotropic process, resulting in the emergence of vortex-like patterns near the exit, shown in Fig. 16(b). This is caused by a delicate balance between the repulsive and attraction force exerted on the agents. Basically, the density of the agents near the exit is greater than that at the periphery. As a consequence, the inner agents (dominated by repulsive force) are inclined to move outward, while the outer agents tend to move toward the desired exit. With

\footnotetext{
${ }^{2}$ Here $\rho_{i}$ is defined as the number of agents within the neighboring volume of the agent $i$ divided by the volume $V=\pi r^{2}=4 \pi r_{0}^{2}$, which is actually the area of a circle of radius $r$ with the center located on $i$. We have checked that other choice of $V$ does not change the qualitative properties of the results presented in Fig. 17, as long as $r$ is far smaller than the linear size of the room
} 

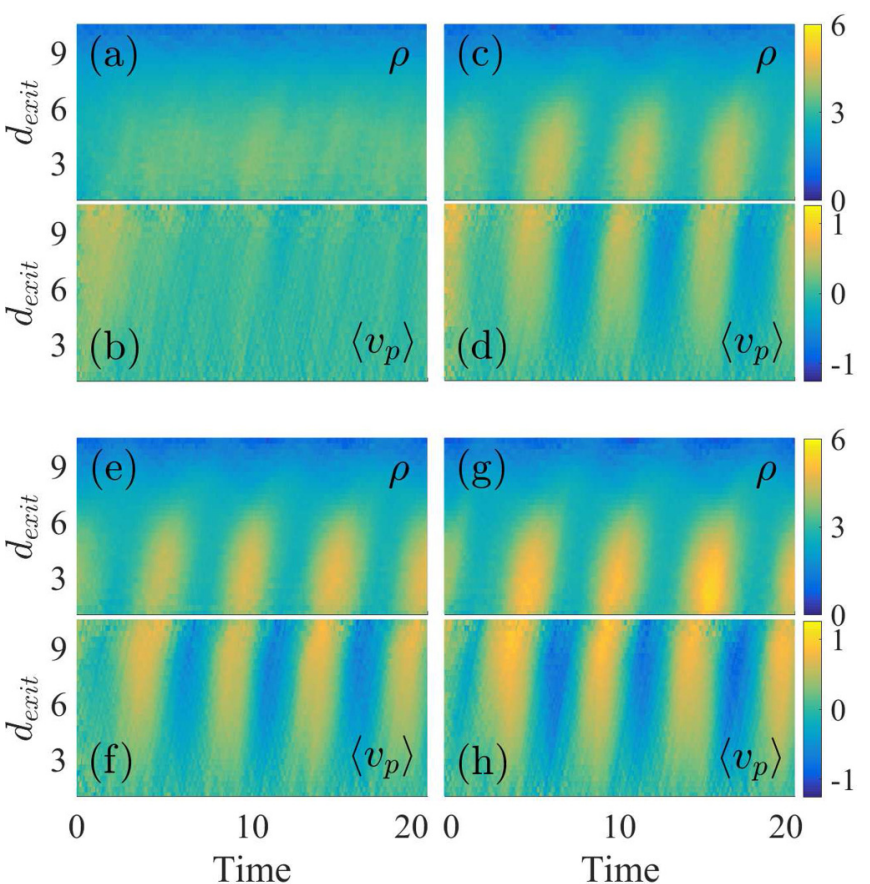

FIG. 17. Spatiotemporal patterns of the local density $\rho$ and the velocity projection of the agents along the direction pointing to the exit for several attraction distances $d_{f}$ and it respectively is $d_{f}=0.5$, 0.8, 1.1, and 1.4 for (a) and (b), (c) and (d), (e) and (f), (g), and (h). The vertical axis represents the distance between agent's position and exit. Periodic like behavior of gathering and bombing of the agents becomes more striking for increasing $d_{f}$.

appropriate conditions, the flow lanes generated by the two distinct groups could form a moment of couple, driving the surrounding agents to form a vertex like pattern. This phenomenon is usually called as traffic waves which is often observed in realistic systems and very likely gives rise to the disaster of stampede accidents $[14,15]$.

When we decrease $d_{f}$ to sufficiently low values, the amplitude of traffic wave becomes vanishing. For instance, for $d_{f}=0.1$, we only find very slight fluctuations in the system, and the agents are keeping calm and move back and forth around the location where they were, see Fig. 16(a) and also the videos in Ref. [50].

We remark that traffic waves is often observed in unidirectional flow scenario with constant width of the road for vehicles, or in the case of evacuation into narrow channel for pedestrians. The emergence of such phenomenon is often ascribed to the lack of and/or the delay of obtaining the surrounding road information. For instance, the individuals at the tail part of a group might push forward without knowing the density of the front part, or drivers can not follow the vehicles in the front of them instantaneously when they suddenly stop or launch and then lead to jamming.

The arise of traffic waves in our model is due to the interplay of the strong desire of the agents to moving to the escaping exit with the influence from local neighbors. Specifically, when uninformed individuals are located in high population density environment, they are more likely to act blindly. For example, once they find some neighbors moving fast, they would think the neighbors are just moving "rightly" to the exit and very likely chase them in blind. The tendency to following the fast pedestrian flow lanes will further attract more surrounding blind individuals to join. Once such chain reaction takes place, the process will hardly stop, and continue until excessive gathering brings huge interior pressure, leading to collapse.

Finally, it is worth mentioning that in two recent experimental studies [51,52], the real pedestrian crowds usually form a bubble- or drop-like pattern instead of the heaplike one observed here when they are passing a narrow bottleneck. We argue that the difference might due to the different movement scenarios investigated: a situation of low competitiveness of the pedestrians (moderate/low pedestrian density with fast moving speed) is considered in [51,52], while we have mainly studied a case of high competitiveness of the pedestrians (quite high pedestrian density circumstance with slow collectively moving speed). In such more "panic" situation, the heaplike pattern of the pedestrians is generally more likely to emerge [53].

\section{CONCLUSIONS AND DISCUSSIONS}

In this paper, we have studied how the two kinds of interaction mechanisms, say avoiding collision and following the fast pedestrian flow lanes, affect the collective motion behavior of pedestrians in three typical motion scenarios. Analogous to other pedestrian models based on anticipation of relative displacement and velocity, the mutual avoiding interaction between every pair of individuals is the typical driving force for the pedestrians trying to keep safe and ordered motion. When the number of individuals in a conserved column increases, however, every individual has to face a crowded space, where one usually can not obtain enough and useful information about the motion profile in the surrounding circumstances. For this reason, the correctness of any prior estimation or anticipation will be greatly discounted. And as such, following the local crowd with relatively high speed flow will be a reasonable (or the only) choice. Interestingly, the two simple heuristic mechanisms allow us to reproduce typical collective motion patterns observed in the real world, and they seem to paly distinct roles in different pedestrian scenarios.

In the case of bidirectional pedestrian flows in a straight corridor, we find that following the fast moving peer partners is very beneficial in a crowding situation, which will drive the pedestrians into a collective moving entity, wherein the agents just only need to obey the mainstream of motion. In this way, the pedestrians are easily able to form clusters with separate flow lanes, and thereby expected to walk to their destinations efficiently with less collisions.

However, we find that the strong attraction by the fast flow lanes does not always work well in some motion scenarios of the pedestrians. For instance, in the case of crossing flows, when the attraction is too strong (large $d_{f}$ ), we find the squeezing of the width of a queue and the enhancement of stability of pedestrian clusters do not increase their passing efficiency, which on the contrary just gives rise to the wasting of the space of the passing channel. In such case, the individuals are unable to organize themselves into alternate waiting and passing, and they are assembled to moving 
together without compromise, finally leading to crowded jam. Remarkably, the strengthening of avoidance mechanism (i.e., a sufficiently large $d_{s}$ ) will become helpful in such situation, where the deceleration of moving speed contributes to the self-organization of the motion behavior into passingwaiting-passing pattern. In the crossing pedestrian scenario, the desire for quickly moving does not play as much an instructive role as it in the bidirectional flow scenario, where the alternate acceleration and deceleration of the pedestrians is actually dominating. Thus the repulsive force due to the avoidance mechanism will play a decisive role in the crossing flows scenario.

In addition, we also observe the negative side of following the fast moving peer-partners in the case of evacuation dynamics. Our preliminary results indicate that strong attraction sometimes means blind obedience, which is likely to cause harmful pedestrian traffic waves. The arise of traffic waves destroys not only the normal moving rhythm resulting in chaotic motions, but also pushes the system density into extreme nonuniform state so that the distribution of the velocity of the pedestrians changed repeatedly. In such situation, once some individuals fall down accidentally, it will very likely trigger stampede accidents and a series of chain disasters.

Sometimes people need to be assembled into a queue to maximize the utilization of a road or channel just like in the bidirectional flow scenario. However, sometimes strong following behavior will weaken the independent judgment of the individuals on external conditions, which leads them to fall into excessive blind obedience of the collective. The blind crowds with great motion inertia is hardly to rein in complex situations, especially when the crowds has already accomplished gathering and begin to move, and without suitable physical boundaries and interventions their moving behaviors would be irrational and dangerous. According to our findings, in realistic situations, people should keep cautious to estimate to what extent should they follow the major flow, especially in the crowded situation with the lack of correct information of surrounding environments.

Finally, we would like to remark that, though no explicit extrinsic noise is added in our current model to guide the movement of the agents like that in the standard Vicsek model, the adjustment process of their disturbed velocities (due to the anticipation and attraction mechanisms) to desired ones will serve as the role of intrinsic noise, making it possible for the pedestrians to self-organize into various ordered motion patterns.

\section{ACKNOWLEDGMENT}

This work was supported by the National Natural Science Foundation of China (Grant No. 11975111).

\section{APPENDIX: DETERMINATION OF THE PARAMETER $d_{m}$}

We denote by $d_{m}$ the average maximum range of visibility of the agents. If the agent $i$ is (partly) sheltered by the others from the viewpoint of $j$, it will not be recognized by $j$, see Fig. 18. As a consequence, the observer $j$ cannot detect the moving status of $i$ even if they might be quite closer. The parameter $d_{m}$ characterizes therefore the average response

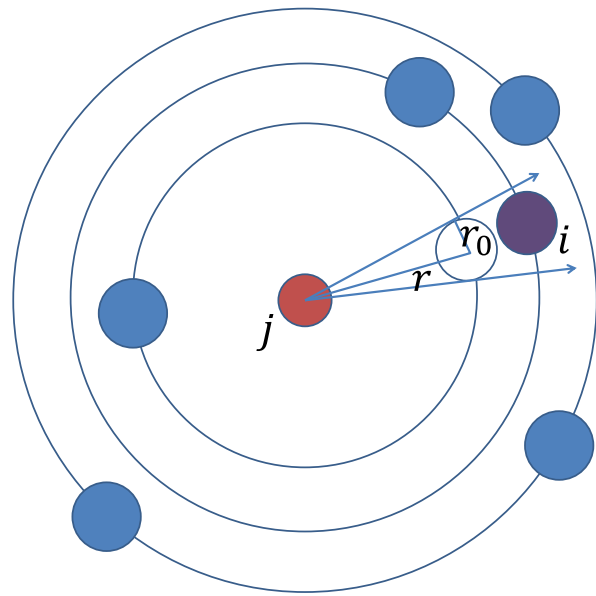

FIG. 18. The view of the focal agent in the center is blocked to some extent by those surroundings, decreasing the accuracy of its responses to neighbors' moving status.

distance of the agents to the moving status of the surrounding neighbors. Obviously, $d_{m}$ is smaller in a dense system than that in a sparse system due to the presence of more shelters.

Here, we give a crude approximation of $d_{m}$. To do this, we first estimate the shelter probability within a distance $d$. In a system with uniformly distributed pedestrians of density $\rho$, away from the position of the observer, the number of agents in the circle with radius $r$ and width $d r$ is $d N(r)=2 \pi \rho r d r$. The probability that the focal agent's view at distance $r$ will be blocked reads as

$$
p(r) d r=\frac{2 \arcsin \left(\frac{r_{0}}{r}\right) d N}{2 \pi} .
$$

Substituting the expression of $d N(r)$, we have approximately $p(r) \approx 2 r_{0} \rho$. The probability that an agent away from the focal agent with distance $d$ can be observed is

$$
P(d)=\exp \left[-\int_{2 r_{0}}^{d}(p(r)) d r\right]=\exp \left(2 r_{0} \rho\left(2 r_{0}-d\right)\right) .
$$

Thus we obtain the relationship between $d$ and $r_{0}$ and $\rho$ as

$$
d=2 r_{0}-\frac{\ln (P(d))}{2 \rho r_{0}} .
$$

In an extreme dense crowd [whose mean density $\rho=\rho_{0} \approx$ $\left.1 /\left(\pi r_{0}^{2}\right)\right]$, the agents can just observe the ones adjacent to them, and we approximate $d_{m}$ as the twice diameter of an agent such that we have

$$
d_{m}=4 r_{0}=2 r_{0}-\frac{\ln \left(P\left(d_{m}\right)\right)}{2 \rho_{0} r_{0}} .
$$

After doing some algebra, we have

$$
\ln \left(P\left(d_{m}\right)\right)=-\frac{4}{\pi} .
$$

For uniform distribution of the pedestrians, the maximum average range of visibility of the agents is then obtained as

$$
d_{m}=2 r_{0}+\frac{2}{\pi \rho r_{0}} \text {. }
$$


Note that in dense pedestrian systems, the vision is more likely to be obscured as compared to that in the case of sparse systems, it is reasonable for us to choose a small $d_{m}$ for dense systems. In our current study, we simply choose $d_{m}$ as 10 and 2 for sparse and dense pedestrian systems, respectively.
[1] T. Butt, T. Mufti, A. Humayun, P. B. Rosenthal, S. Khan, S. Khan, and J. E. Molloy, Myosin motors drive long range alignment of actin filaments, J. Biol. Chem. 285, 4964 (2010).

[2] I. D. Couzin, C. C. Ioannou, G. Demirel, T. Gross, C. J. Torney, A. Hartnett, L. Conradt, S. A. Levin, and N. E. Leonard, Uninformed individuals promote democratic consensus in animal groups, Science 334, 1578 (2011).

[3] I. Karamouzas, B. Skinner, and S. J. Guy, Universal Power Law Governing Pedestrian Interaction, Phys. Rev. Lett. 113, 238701 (2014).

[4] T. J. Pitcher and C. J. Wyche, Predator-avoidance behaviours of sand-eel schools: why schools seldom split, in Predators and Prey in Fishes: Proceedings of the 3rd Biennial Conference on the Ethology and Behavioral Ecology of Fishes, Held at Normal, Illinois, U.S.A., May 19-22, 1981, edited by D. L. G. Noakes, D. G. Lindquist, G. S. Helfman, and J. A. Ward (Springer, Netherlands, Dordrecht, 1983) pp. 193-204.

[5] W. Herrnkind, Queuing behavior of spiny lobsters, Science 164, 1425 (1969).

[6] R. G. Bill and W. F. Herrnkind, Drag reduction by formation movement in spiny lobsters, Science 193, 1146 (1976).

[7] A. Gorrini, L. Crociani, C. Feliciani, P. Zhao, K. Nishinari, and S. Bandini, Social groups and pedestrian crowds: experiment on dyads in a counter flow scenario, arXiv: 1610.08325.

[8] C. Feliciani and K. Nishinari, Empirical analysis of the lane formation process in bidirectional pedestrian flow, Phys. Rev. E 94, 032304 (2016).

[9] R. van Drongelen, A. Pal, C. P. Goodrich, and T. Idema, Collective dynamics of soft active particles, Phys. Rev. E 91, 032706 (2015).

[10] H. Levine, W.-J. Rappel, and I. Cohen, Self-organization in systems of self-propelled particles, Phys. Rev. E 63, 017101 (2000).

[11] T. Vicsek, A. Czirók, E. Ben-Jacob, I. Cohen, and O. Shochet, Novel Type of Phase Transition in a System of Self-Driven Particles, Phys. Rev. Lett. 75, 1226 (1995).

[12] T. Vicsek and A. Zafeiris, Collective motion, Phys. Rep. 517, 71 (2012).

[13] D. Helbing and P. Molnár, Social force model for pedestrian dynamics, Phys. Rev. E 51, 4282 (1995).

[14] D. Helbing, A. Johansson, and H. Z. Al-Abideen, Dynamics of crowd disasters: An empirical study, Phys. Rev. E 75, 046109 (2007).

[15] W. Yu and A. Johansson, Modeling crowd turbulence by manyparticle simulations, Phys. Rev. E 76, 046105 (2007).

[16] D. Helbing, L. Buzna, A. Johansson, and T. Werner, Selforganized pedestrian crowd dynamics: Experiments, simulations, and design solutions, Transp. Sci. 39, 1 (2005).

[17] A.-H. Olivier, A. Marin, A. Crétual, and J. Pettré, Minimal predicted distance: A common metric for collision avoidance during pairwise interactions between walkers, Gait Posture 36, 399 (2012).
[18] D. R. Parisi, P. A. Negri, and L. Bruno, Experimental characterization of collision avoidance in pedestrian dynamics, Phys. Rev. E 94, 022318 (2016).

[19] M. Moussaïd, D. Helbing, S. Garnier, A. Johansson, M. Combe, and G. Theraulaz, Experimental study of the behavioural mechanisms underlying self-organization in human crowds, Proc. R. Soc. B 276, 2755 (2009).

[20] J. L. Silverberg, M. Bierbaum, J. P. Sethna, and I. Cohen, Collective Motion of Humans in Mosh and Circle Pits at Heavy Metal Concerts, Phys. Rev. Lett. 110, 228701 (2013).

[21] D. C. Duives, W. Daamen, and S. P. Hoogendoorn, State-ofthe-art crowd motion simulation models, Transp. Res. Part C: Emerging Technol. 37, 193 (2013).

[22] R. L. Hughes, A continuum theory for the flow of pedestrians Transp. Res. Part B: Methodol. 36, 507 (2002).

[23] L. P. Kadanoff, Simulating hydrodynamics: A pedestrian model, J. Stat. Phys. 39, 267 (1985).

[24] S. Hoogendoorn and P. H. L. Bovy, Gas-Kinetic Modeling and Simulation of Pedestrian Flows, Transp. Res. Rec. 1710, 28 (2000).

[25] S. P. Hoogendoorn, F. van Wageningen-Kessels, W. Daamen, D. C. Duives, and M. Sarvi, Continuum theory for pedestrian traffic flow: Local route choice modelling and its implications, Transp. Res. Part C: Emerging Technol. 59, 183 (2015).

[26] S. P. Hoogendoorn, F. L. van Wageningen-Kessels, W. Daamen, and D. C. Duives, Continuum modelling of pedestrian flows: From microscopic principles to self-organised macroscopic phenomena, Physica A 416, 684 (2014).

[27] K. Nagel and M. Schreckenberg, A cellular automaton model for freeway traffic, J. Phys. I France 2, 2221 (1992).

[28] W. G. Weng, T. Chen, H. Y. Yuan, and W. C. Fan, Cellular automaton simulation of pedestrian counter flow with different walk velocities, Phys. Rev. E 74, 036102 (2006).

[29] Y. F. Yu and W. G. Song, Cellular automaton simulation of pedestrian counter flow considering the surrounding environment, Phys. Rev. E 75, 046112 (2007).

[30] D. Helbing, I. Farkas, and T. Vicsek, Simulating dynamical features of escape panic, Nature 407, 487 (2000).

[31] W. J. Yu, R. Chen, L. Y. Dong, and S. Q. Dai, Centrifugal force model for pedestrian dynamics, Phys. Rev. E 72, 026112 (2005).

[32] M. Chraibi, A. Seyfried, and A. Schadschneider, Generalized centrifugal-force model for pedestrian dynamics, Phys. Rev. E 82, 046111 (2010).

[33] A. Johansson, D. Helbing, and P. K. Shukla, Adv. Complex Syst. 10, 271 (2007)

[34] D. Helbing and A. Johansson, Encyclopedia of Complexity and Systems Science, edited by R. A. Meyers (Springer, New York, 2009), pp. 6476-6495.

[35] F. Zanlungo, T. Ikeda, and T. Kanda, Social force model with explicit collision prediction, Europhys. Lett. 93, 68005 (2011). 
[36] A. Johansson and D. Helbing, in Pedestrian and Evacuation Dynamics 2008, edited by W. W. F. Klingsch, C. Rogsch, A. Schadschneider, and M. Schreckenberg (Springer, Berlin, Heidelberg, 2010), pp. 203-214.

[37] R. Raghavendra, A. Del Bue, M. Cristani, and V. Murino, Abnormal crowd behavior detection by social force optimization, in Human Behavior Understanding, edited by A. A. Salah and B. Lepri (Springer, Berlin, Heidelberg, 2011), pp. 134-145.

[38] D. R. Parisi, M. Gilman, and H. Moldovan, A modification of the Social Force Model can reproduce experimental data of pedestrian flows in normal conditions, Physica A 388, 3600 (2009).

[39] P. Degond, C. Appert-Rolland, M. Moussaïd, J. Pettré, and G. Theraulaz, A hierarchy of Heuristic-based models of crowd dynamics, J. Stat. Phys. 152, 1033 (2013).

[40] D. Strömbom, Collective motion from local attraction, J. Theor. Biol. 283, 145 (2011).

[41] D. Grossman, I. S. Aranson, and E. Ben Jacob, Emergence of agent swarm migration and vortex formation through inelastic collisions, New J. Phys. 10, 023036 (2008).

[42] L. Barberis and F. Peruani, Large-Scale Patterns in a Minimal Cognitive Flocking Model: Incidental Leaders, Nematic Patterns, and Aggregates, Phys. Rev. Lett. 117, 248001 (2016).

[43] P. A. Langston, R. Masling, and B. N. Asmar, Crowd dynamics discrete element multi-circle model, Saf. Sci. 44, 395 (2006).

[44] H. Singh, R. Arter, L. Dodd, P. Langston, E. Lester, and J. Drury, Modelling subgroup behaviour in crowd dynamics DEM simulation, Appl. Math. Modell. 33, 4408 (2009).
[45] J. Kwak, H.-H. Jo, T. Luttinen, and I. Kosonen, Jamming transitions induced by an attraction in pedestrian flow, Phys. Rev. E 96, 022319 (2017).

[46] N. A. Morton and S. C. Hendy, Symmetry breaking in pedestrian dynamics, arXiv:1605.05437.

[47] D. Helbing, I. J. Farkas, P. Molnar, and T. Vicsek, Simulation of pedestrian crowds in normal and evacuation situations, Pedestrian and Evacuation Dynamics, Vol. 21 (Springer, Berlin, 2002), pp. 21-58

[48] A. Bottinelli, D. T. J. Sumpter, and J. L. Silverberg, Emergent Structural Mechanisms for High-Density Collective Motion Inspired by Human Crowds, Phys. Rev. Lett. 117, 228301 (2016).

[49] S. J. Guy, S. Curtis, M. C. Lin, and D. Manocha, Least-effort trajectories lead to emergent crowd behaviors, Phys. Rev. E 85, 016110 (2012).

[50] See Supplemental Material at http://link.aps.org/supplemental/ 10.1103/PhysRevResearch.2.043250 for typical videos of the pedestrians.

[51] A. Nicolas, S. Bouzat, and M. N. Kuperman, Pedestrian flows through a narrow doorway: Effect of individual behaviours on the global flow and microscopic dynamics, Transp. Res. Part B: Methodol. 99, 30 (2017).

[52] X. Shi, Z. Ye, N. Shiwakoti, D. Tang, and J. Lin, Examining effect of architectural adjustment on pedestrian crowd flow at bottleneck, Physica A 522, 350 (2019).

[53] J. M. Pastor, A. Garcimartín, P. A. Gago, J. P. Peralta, C. MartínGómez, L. M. Ferrer, D. Maza, D. R. Parisi, L. A. Pugnaloni, and I. Zuriguel, Experimental proof of faster-is-slower in systems of frictional particles flowing through constrictions, Phys. Rev. E 92, 062817 (2015). 